\title{
ON THE SINGULARITIES OF MASSIVE SUPERSTRING AMPLITUDES
}

\author{
Omar FODA \\ Institute for Theoretical Physics, Princetonplein 5, P.O. Box 80006, 3508 TA Utrecht, The Netherlands
}

Received 12 February 1987

\begin{abstract}
Superstring one-loop amplitudes with massive external states are shown to be in general ill-defined due to internal on-shell propagators. However, we argue that since any massive string state (in the uncompactified theory) has a finite lifetime to decay into massless particles, such amplitudes are not terms in the perturbative expansion of physical $S$-matrix elements: these can be defined only with massless external states. Consistent massive amplitudes require an off-shell formalism.
\end{abstract}

1. It is generally expected that superstring theories [1] are totally finite. Complete expressions of multiloop amplitudes are not yet available [2], but explicit computations of one-loop amplitudes with massless external states are straightforward [1]. They exhibit the singularities expected in a narrow-resonance approximation [3], where unitarity is implemented perturbatively, and otherwise are completely finite. In particular, the tadpole divergence associated with the transition of a massless dilaton into the vacuum is absent, due to the supersymmetry of the spectrum.

In these computations certain potential divergences are suppressed by the vanishing of the oneloop self-energies of the massless external states. As mentioned in ref. [4], this is no longer true for massive external states, whose self-energies are not protected from radiative correlations by gauge or supersymmetry. Our purpose here is to discuss what happens in this case. As an example we obtain a simple one-loop amplitude with 1 massive and 2 massless external states, through factorizing an amplitude with 4 massless states. It is shown to be divergent due to a massive propagator that must be evaluated on shell due to the kinematics, and cannot be taken off-shell in an $S$-matrix formalism. On the other hand, we remark that any massive string state, that is onshell in tree-approximation sense, can decay into a set of on-shell massless states. Thus massive states have finite lifetimes and cannot be used as asymptotic states. (In this work, "massive" and "massless" is meant in terms of the original uncompactified the- ory. Upon compactification one can generate stable massive solitons, they will be discussed separately below, with no change in conclusions.)

Consequently, we take the viewpoint that string amplitudes with massive external states are not terms in the perturbative expansion of any physical $S$ matrix element. These can be defined only with massless external states; they have been shown to be one-loop finite, and may remain so at higher orders.

2. To begin, we recall the form of a typical massless superstring one-loop amplitude. Consider the scattering of 4 massless states in type-II theory. A standard computation outlined in ref. [1] gives

$A[1,2,3,4]=K[1,2,3,4] \int_{F} \mathrm{~d}^{2} \tau[\operatorname{Im} \tau]^{-2} F_{\mathrm{s}}(\tau)$,

where

$$
\begin{aligned}
& F_{\mathrm{s}}(\tau)=[\kappa \pi]^{4}[\operatorname{Im} \tau]^{-3} \int \prod_{I=1}^{3} \mathrm{~d}^{2} \nu_{I} \prod_{I=1}\left[\chi_{I J}\right]^{k_{I} \cdot k_{J} / 2}, \\
& \chi_{I J}=2 \pi \exp \left(\frac{-\pi\left(\operatorname{Im} \nu_{I J}\right)^{2}}{\operatorname{Im} \tau}\right)\left|\frac{\theta_{1}\left(\nu_{I I} \mid \tau\right)}{\theta_{1}^{\prime}(0 \mid \tau)}\right| \\
& \nu_{J I}=\nu_{J}-\nu_{I}
\end{aligned}
$$

In analogue-model terms [5]; $\nu_{J}(J=1,2,3)$ parametrizes the position of 3 sources (in general dipoles or higher multipoles depending on the vertex) of strength $k_{J}$ : the external momentum at vertex $J$, rel- 
ative to one located at $\tau$ on a two-dimensional homogeneous toroidal conductor. $\tau$ parametrizes the shape of the torus, and $K[1,2,3,4]$ is a kinematical factor that contains the wavefunctions of the external states. For notation and conventions, we follow ref. [1]; in particular our metric is $[-,+, \ldots,+]$. Singularities occur in (1) at configurations where two or more sources coincide. At this point one has to distinguish between (a) singularities that occur only for specific values of external momenta, and (b) singularities that make the amplitude ill-defined irrespective of the external momenta. The former are poles of on-shell intermediate states, and are perfectly acceptable in a formalism based on a narrow resonance approximation. Inclusion of higher order radiative corrections will ameliorate them. But the latter are genuine divergences: if they show up in a physical quantity they require a subtraction procedure.

Let us consider an example of the first type. In eq. (1) take $\nu_{12}=\left|\nu_{1}-\nu_{2}\right| \sim \epsilon \rightarrow 0$. In this limit $\chi_{I J}\left[\nu_{I J}, \tau\right] \sim 2 \pi \epsilon$, and we obtain

$F_{\mathrm{s}}(\tau) \sim \int_{0} \mathrm{~d}^{2} \epsilon(\epsilon)^{k_{1} \cdot k_{2} / 2}$,

where we neglect a prefactor, containing all other variables, that is irrelevant for the discussion. For

$k_{1} \cdot k_{2} / 2=N$,

where $N$ is a negative integer $\leqslant-2,(2)$ is divergent.

In our space-like metric, $N$ is the invariant [mass] ${ }^{2}$ in the relevant channel, and singularities coincide with tuning the external momenta to excite a resonance. Now let us go back to (1), and consider the integration region where the $3 \nu_{l}$ lie within an infinitesimal ball of radius $\epsilon$. Then

$F_{\mathrm{s}}(\tau) \sim \int_{0} \mathrm{~d}^{2} \epsilon(\epsilon)^{\left(k_{1} \cdot k_{2}+k_{1} \cdot k_{3}+k_{2} \cdot k_{3}\right) / 2}$.

As all external particles are massless we obtain

$\sum_{I<J} k_{I} \cdot k_{J}=0 \rightarrow k_{1} \cdot k_{2}+k_{1} \cdot k_{3}+k_{2} \cdot k_{3}=0$,

and the potential divergence disappears. A similar situation takes place in heterotic-string computations [4]. The limit $\epsilon \rightarrow 0$ in (3) corresponds to a configuration where the torus sits on an external massless propagator, and acts as a self-energy radiative correction. That such configurations give vanishing contributions is an aspect of the nonrenormalization of the self-energies of massless states. They are protected by gauge and/or supersymmetries.

However, if the external particles were not all massless, (4) would not be true, and (3) need not be regular. For example, if particle 4 , located at $\tau$ is massive, conservation of momentum will force the invariant mass in the $\{1,2,3\}$ channel to be equal to that of 4 , and (3) will be singular. Since the singularity will be present for all allowed external momenta, this would be an example of type (b) mentioned above, and the amplitude would be illdefined.

3. Let us work out a simple example. Consider a one-loop amplitude in type-II superstring theory with 2 massless and 1 massive external states. (We will choose an amplitude which is common to both types IIA and II B.) It is straightforward to compute this direclty in light-cone operator formalism: the computation parallels that of massless amplitudes. The only new ingredient is the massive vertex. It factorizes into functions of left- and right-moving modes. Restricting to transverse polarization, which is allowed for a small number of external legs, we obtain as a simple example

$V(\xi, k, \tau)=g \xi_{i j \widetilde{k l}} B^{i j} \widetilde{B}^{\widetilde{k l}} \exp [\mathrm{i} k \cdot x(\tau)]$,

where

$$
\begin{aligned}
& B^{i j}(\tau)=B^{i}(\tau) B^{j}(\tau) \\
& \quad=\left[P^{i}(\tau)+k_{m} R^{i m}(\tau)\right]\left[P^{j}(\tau)+k_{n} R^{i n}(\tau)\right],
\end{aligned}
$$

$R^{i j}(\tau)=\frac{1}{8} \bar{S}(\tau) \gamma^{i j-} S(\tau)$,

with similar expressions for $\widetilde{B}^{\widetilde{k l}} \cdot \xi_{i j \widehat{k l}}$ contains all polarization vectors, and the choice of contractions and symmetrizations determines the specific state considered. All other notations are standard [1]. Notice that (5) contains a factor quartic in the fermion zero-modes. Hence an amplitude with two massless and a single massive vertex of type (5) will contain $8 S_{0}$ (and $8 \widetilde{S}_{0}$ ) operators and will therefore be nonvanishing, in general.

A more direct way to obtain the required ampli- 
tude is to factorize a four-particle massless amplitude on the required massive state. Consider (1) in the limit (2), and factorize it on a massive scalar which we denote by 5 . [ In general there will be more than 1 scalar, depending on the way we contract the polarization tensors of the initial massless particles, but our discussion is valid for any of them.] Schematically, we have

$A_{\text {1-loop }}[1,2,3,4]$

$$
\rightarrow A_{\text {1-loop }}[1,2,5] \text { [ scalar-pole] } A_{\text {tree }}[5,3,4] \text {. }
$$

Picking up the residue on the RHS of (6), which is well-defined if 1,2 and 5 are not overlapping, and factorizing at the constant three-point function $A_{\text {tree }}[5,3,4]$ we obtain the desired amplitude. Let us obtain the [level-1 massive scalar $\rightarrow$ two-graviton]amplitude (ground states are denoted as level-0) from four-graviton amplitude. For convenience we rewrite (1) in the more symmetric form [1]

$$
\begin{aligned}
& A_{1-\text { loop }}[1,2,3,4]=K[1,2,3,4] \\
& \quad \times \int\left(\prod_{I=1}^{4} \mathrm{~d}^{2} z_{I}\right)|\omega|^{-2}\left(\frac{-4 \pi}{\ln |\omega|}\right)^{5} \\
& \times \prod_{I<J}\left[\chi_{I J}\right]^{k_{I} \cdot k_{J} / 2},
\end{aligned}
$$

where $\omega=\prod_{i=1}^{4} z_{i}$. Factorizing on a scalar pole, the kinematic factor $K[1,2,3,4]$ with all polarization tensors will also factorize with no index contractions between the two factors. Fixing the position of $z_{4}$ and taking the limit $z_{3} \rightarrow z_{4}$, we obtain the measure

$$
\prod_{I=1}^{4} \mathrm{~d} z_{I} \rightarrow \mathrm{d} z_{1} \mathrm{~d} z_{2} \mathrm{~d} z_{5}
$$

where we have relabelled the overlap point by 5 for clarity, since a different particle is now attached to it. Similarly

$$
1 /|\omega|^{2} \rightarrow 1 /\left|z_{1} z_{2} z_{5}^{2}\right|, \quad \ln |\omega| \rightarrow \ln \left|z_{1} z_{2} z_{5}^{2}\right|,
$$

and

$$
\begin{aligned}
& \prod_{I<J}\left[\chi_{I J}\right]^{k_{l} \cdot k_{J} / 2} \rightarrow\left[\chi_{12}\right]^{k_{1} \cdot k_{2} / 2}\left[\chi_{15}\right]^{k_{2} \cdot k_{5} / 2} \\
& \quad \times\left[\chi_{25}\right]^{k_{2} \cdot k_{s} / 2}\left[“ \chi_{55} "\right]^{k_{3} \cdot k_{4} / 2}
\end{aligned}
$$

where we have used $k_{5}=k_{3}+k_{4}$, and " $\chi_{55}$ " stands for $\chi_{34}$ in the limit $\left|z_{3}-z_{4}\right|=\epsilon \rightarrow 0$, namely $2 \pi|\epsilon|$. From $\left(k_{3}+k_{4}\right)^{2}=k_{5}^{2}=-8=2 k_{3} \cdot k_{4}$, where the normalization in ref. [1] is used we recover the scalar pole (6), and can now pick up the residue.

Next, we have to factor out the [massive scalar /two-graviton] coupling. Once again, this can be computed either directly, or from the general threesupersymmetric-reggeon coupling [6], and the fact that a three-pomeron vertex is a product of two threereggeon vertices with suitable symmetrizations. However, the easiest way to obtain it is once again through factorization of a suitable amplitude on the required state. This time we factorize the four-graviton tree-amplitude. In type-II superstring theory the tree amplitude for four-massless boson scattering is

$$
\begin{gathered}
A_{\text {tree }}(1,2,3,4)=\frac{\kappa^{2}}{128} \frac{\Gamma(-s / 8) \Gamma(-t / 8) \Gamma(-u / 8)}{\Gamma(1+s / 8) \Gamma(1+t / 8) \Gamma(1+u / 8)} \\
\quad \times \xi_{1}^{\mu_{1} \mu_{2}} \xi_{2}^{\nu_{1} \nu_{2}} \xi_{3}^{\rho_{1} \rho_{2}} \xi_{4}^{\lambda_{1} \lambda_{2}} K_{\mu_{1} \nu_{1} \rho_{1} \lambda_{1}} \tilde{K}_{\mu_{2} \nu_{2} \rho_{2} \lambda_{2}}
\end{gathered}
$$

in the notation of ref. [1].

To obtain external gravitons, we symmetrize the polarization tensors. Noting that the $K$-factors contain four-momenta each, and taking the limit $s \rightarrow 8$, $t+u \rightarrow-8$ (preserving the constraint $s+t+u=0$ ), the $\Gamma$-functions give the required level-one pole: $c / \epsilon$, where $c$ is momentum independent. The residue is $\sim$ [level-one state $\rightarrow$ two gravitons $]^{2}$. For the level-one state to be a scalar we have to factorize the kinetic factors appropriately: the indices of the particle sets $\{1,2\}$ and $\{3,4\}$ should not mix; there should be no cross contractions. Going through the above steps, the coupling turns out to have a linear form that covariantizes to $g \phi R^{\mu \nu \rho \sigma} R_{\mu \nu \rho \sigma}$, where $\phi$ is the massive scalar, and $g$ is a constant. The point is that the momenta that give the derivatives in the Riemann tensor come solely from kinematic factors $K$. No extra momenta come from the expansion of the $\Gamma$-functions. The latter contribute only to the overall constant $g$.

Thus, aside from an overall constant that is irrelevant for our discussion of the singularities, factoring out the three-point tree amplitude from the residue of (6) amounts to factoring out the corresponding kinematic factor. Up to a normalization we are left with 


$$
\begin{aligned}
& A_{\text {1-loop }}[1,2,5]=K[1,2,5] \widetilde{K}[1,2,5] \\
& \times \int\left(\prod_{I=1,2,5} \mathrm{~d}^{2} z_{I}\right) \\
& \times \frac{1}{\left|z_{1} z_{2} z_{5}^{2}\right|^{2}} \ln \left|z_{1} z_{2} z_{5}^{2}\right| \\
& \quad \times\left[\chi_{12}\right]^{k_{1} \cdot k_{5} / 2}\left[\chi_{15}\right]^{k_{1} \cdot k_{5} / 2}\left[\chi_{25}\right]^{k_{1} \cdot k_{5} / 2},
\end{aligned}
$$

where

$$
K[1,2,5]=\left[k_{1}^{i} \xi_{1}^{j} k_{2}^{k} \xi_{2}^{i}\right]\left[\delta_{i j} \delta_{j l}-\delta_{i l} \delta_{j k}\right]
$$

and similarly for $\tilde{K}[1,2,5]$.

Let us check (9) for singularities. In the limit $\left|z_{1}-z_{2}\right| \sim \epsilon \rightarrow 0, \chi_{12} \sim \epsilon,\left(k_{1}+k_{2}\right)^{2}=2 k_{1} \cdot k_{2}=k_{5}^{2}=-8$, and we obtain

$$
\int\left[\mathrm{d}^{2} \epsilon\right][\epsilon]^{k 1 \cdot k_{2} / 2} \log \epsilon .
$$

This singularity is momentum-independent, and the amplitude is ill-defined. It is easy to see that the original amplitude with 4 massless states had configurations with double poles corresponding to both $\left\{\nu_{3}, \nu_{4}\right\}$ and $\left\{\nu_{1}, \nu_{2}\right\}$ coming together. Factorizing on one of these, as we did above, leaves us with a residue that, not only contains the other pole, but also satisfies kinematical conditions such that that pole cannot be evaded by any choice of external momenta. Obviously, this is a formal problem, directly related to the limitations of the on-shell formalism that we are using. If one worked with consistent off-shell amplitudes, then there may be a way to define the massive $S$-matrix elements through a limiting procedure. However, as we will argue below, even this is unnecessary. Let us, for the moment, accept the problem, and consider its implications. (The situation in bosonic strings is definitely more serious. For example, the integrand of the one-loop amplitude for the extended closed bosonic string contains the extra factor

$$
C[\tau]=4\left(\frac{1}{2} \ln \tau\right)^{-12} \mathrm{e}^{4 \pi \operatorname{Im} \tau}\left|f\left(\mathrm{e}^{2 \pi \mathrm{i} \tau}\right)\right|^{-48},
$$

which leads to a divergence in the limit $\tau \rightarrow \infty$, which is within the fundamental region of integration. This leads to the well-known tachyon and dilaton divergences, that are absent in superstrings. They cannot be eliminated by any choice of external momenta, or even by analytic continuations off-shell, and thus are more intrinsic.)

4. What is the significance of these divergences? Since they are related to the on-shellness of the external massive states, one may consider - as mentioned above - that an off-shell formulation may be the starting point to obtain well-defined expressions, separate the would-be divergences, then add suitable counterterms to cancel them. (A complete off-shell formalism is not available yet, though progress have been made in that directions [7,8].) On the other hand, loop amplitudes are presumably terms in a perturbative expansion of $S$-matrix elements. Their relevance is solely derived from that. What we will point out now, is that all massive string states have finite lifetimes. Thus they cannot be considered as asymptotic states in the limit $t \rightarrow \pm \infty$, and one cannot define physical $S$-matrix elements with them as external states. They always have to be produced at finite $t$, by sources that are suitable functions of massless states. Only the latter can be produced or detected asymptotically. Since no physical massive $S$-matrix elements exist, we adopt the viewpoint that whether terms in the corresponding perturbative expansion are well-defined or not is irrelevant to the consistency of the theory.

The reason why any massive string state is unstable follows also directly from tree-amplitude factorization [9]: starting from a general massless tree amplitude with a sufficiently large number of arbitrary massless states, one can factorize on any on-shell massive state, given the appropriate choice of external momenta and spin (contractions of polarization tensors) along the factorized channel

$$
\begin{aligned}
& A[1, \ldots, N] \\
& \quad \rightarrow B[1, \ldots m, \text { massive particle] [massive pole] } \\
& \quad \times C[\text { massive particle, } m+1, \ldots, N] .
\end{aligned}
$$

This is of course the method originally used by Scuito [10] to obtain the coupling between any 3 string states: the three-reggeon coupling. It agrees with the computation based on the spectrum generating algebra [11]. It can be extended to the $N$-reggeon coupling and to fermionic strings. (For a recent discussion and references see refs. [18,12].) To 
summarize, any string state corresponds to a pole in the propagator that appears in tree computations, and with a suitable choice of external momenta and spin it can be excited and factorized on. The RHS of (11) defines an amplitude for the decay of a massive state into a number of massless states. Thus there is always a radiative-correction (not necessarily of one-loop order) of the on-shell propagator of that massive state, that can be cut on on-shell intermediate states, and thus has a finite imaginary part, the position of the pole in the full propagator is off the real axis, and the massive state has a finite lifetime: it cannot be an asymptotic state.

Now, consider a scattering process where a massive particle is produced with sufficiently large velocity that it travels far enough to be detected. Usually, one would think of it as stable, and proceed to compute $S$-matrix elements. However, since this is an approximation, there is no reason to expect string theory to yield meaningful results in such computations unless the rules of string perturbation theory are also correspondingly modified. The correct way to do things would be to account for the massive particle being off-shell and use the appropriate off-shell vertices. (Conformally-invariant treeamplitudes with off-shell external states and ghost contributions have been obtained in ref. [8].)

Another way of saying the same thing is that, if one could sum over string perturbation theory, then the intermediate massive poles would be smoothed out to peaks. Factorizing over these would lead to amplitudes with off-shell external states. These would be well defined. Such problems appear also in field theory. But there we know how to deal with them, since we know - at least in simple cases - how to sum over certain classes of Feynman diagrams, obtain the renormalized propagator with a shifted pole, etc. In string theory, there is - as yet - no simple way to do that.

Finally, we remark that ambiguities in massive amplitudes are also present at the tree-level: tree amplitudes with both massive and dilaton states are ill-defined in the soft-dilaton limit [13]. The ambiguity comes from configurations where the soft-dilaton sits on an external massive propagator. In the soft-dilaton limit (vanishing dilaton momentum) the massive propagator connecting the soft-dilaton with the rest of the diagram is evaluated on-shell. This does not happen with massless propagators since the dilaton couples to the trace of the energy-momentum tensor which vanishes for on-shell massless particles.

5. In the course of this work, we found that these singularities have been discussed in the literature. In ref. [9], it was suggested that they may be renormalized, or that one should restrict oneself to evaluating massless amplitudes, but no rationale for this was given. In ref. [14], summation of string perturbation series, on the same lines as mentioned above was also suggested and qualitatively outlined. More recently, Weinberg has also discussed these singularities [15]. His proposal is to renormalize them. $\mathrm{He}$ finds that the requirements of consistent factorization provide sufficient conditions to determine the finite parts of the renormalized amplitudes. Thus one does not require separate renormalization conditions, and the usual finite ambiguities of a renormalization procedure would not appear here. However, his renormalized vertex operators with radiatively-corrected mass-shells do not lead to conformally-invariant expressions when used to compute, e.g., tree-amplitudes. (The reason may well be that ghost contributions were not included.) As should be clear from our discussion, these singularities are an artifact of the formalism. If one wishes to discuss the scattering of massive states one has to abandon the on-shell formulation. In a proper offshell formalism there should be no inconsistencies.

Finally, we briefly discuss what to expect in models with stable massive particles. That would obviously depend on how the mass is generated. The only such states we know are the solitons in torus compactifications. These could be treated as ordinary states with quantized momenta in certain directions, and the same remarks would apply to them: all excited states (these with internal oscillator excitations) are unstable and decay to lowest mass states in the same sector. Only the latter can be asymptotic states. They are massless states of the original non-compactified theory, with non-vanishing momenta, and the nonrenormalization theorems apply to them, suppressing potential divergences. Notice that all such solitions have Planck-scale masses: modular invariance requires partition functions with tree-approximation masses equally-spaced in planck units. Realistic masses must have a different origin. The point is that 
a partition function gives the spectrum of the bare "one-string" theory, and one expects "many-string" effects in a finite, but broken supersymmetric theory to give finite mass-shifts and a more realistic spectrum. If that is the way realistic small masses are generated, then loop computations, as given above, would still be meaningful only with massless external states: many-string effects would show up only in summations over perturbation theory.

I wish to thank P. Jetzer for bringing ref. [15] to my attention, H.B. Nielsen and E. Verlinde for useful remarks, and in particular B. de Wit for many discussions, and for reading the manuscript. This work was supported by the "Stichting voor Fundamenteel Onderzoek der Materie (F.O.M.)"

\section{References}

[1] J.H. Schwarz, Phys. Rev. 89 (1982) 223, and references therein.

[2] L. Alvarez-Gaumé and P. Nelson, in: Supersymmetry, supergravity and superstrings ' 86 (World Scientific, Singapore, 1976), and references therein.
[3] M. Jacob, ed., Dual theory, Physics Reports reprint Vol. I, (North Holland, Amsterdam, 1974).

[4] D. Gross et al., Nucl. Phys. B 267 (1986) 75.

[5] H.B. Nielsen, Nordita preprint (1969); D. Fairlie and H.B. Nielsen, Nucl. Phys. B 20 (1970) 637.

[6] M.B. Green and J.H. Schwarz, Nucl. Phys. B 198 (1982) 441.

[7] M. Bershadski, Intern. J. Mod. Phys. A1 (1986) 443; A. Cohen, G. Moore, P. Nelson and J. Polchinski, Nucl. Phys. B 281 (1987) 127.

[8] P. Di Vecchia, R. Nakayama, J.L. Petersen, S. Sciuto and J. Sidenius, Nordita preprints (1986).

[9] S. Mandelstam, Phys. Rep. 13 (1974) 259, and references therein.

[10] S. Sciuto, Lett. Nuovo Cimento 2 (1969) 411.

[11] M. Ademello, E. Del Giudice, P. Di Vecchia and S. Fubini, Nuovo Cimento A 19 (1974) 181.

[12] P. Di Vecchia, R. Nakayama, J.L. Petersen and S. Sciuto, Nucl. Phys. B 282 (1987) 103.

[13] M. Ademello et al., Nucl. Phys. B 94 (1975) 221.

[14] V. Alessandrini, D. Amati, M. Le Bellac and D. Olive, Phys. Rep. C 1 (1971) 269.

[15] S. Weinberg, Texas preprint UTTG-22-85. 